\title{
ON THE APPLICATION OF TIME-REVERSED SPACE-TIME BLOCK CODE TO AERONAUTICAL TELEMETRY
}

\author{
Michael Rice \\ Brigham Young University \\ Md. Shah Afran \\ Mohammad Saquib \\ The University of Texas at Dallas
}

\begin{abstract}
Generalized time-reversed space-time block codes (GTR-STBC) are introduced as a conceptual tool to examine the impact of unequal power allocation in aeronautical telemetry channels. Two transmitting antennas are employed to exploit partial channel state information. GTR-STBC are observed to perform the best trade-off between the signal-to-noise ratio and inter symbol interference. It is also observed that the optimum transmitter power profile for the measured channel is significantly different than that in the statistical channel model.
\end{abstract}

\section{INTRODUCTION}

Aeronautical telemetry deals with a wideband air-to-ground communication link that usually comprises of frequency selective fading channels. In addition, severe size, weight and power limitations are imposed on the airborne transmitter. Highly power-efficient transmitters, such as radio frequency (RF) power amplifiers operating in full saturation, and constant-envelope modulation schemes are employed to overcome these limitations. Since frequency selective fading leads to inter-symbol interference (ISI), techniques for mitigating ISI using the aeronautical telemetry standard IRIG 106 [1] (especially the shaped offset quaternary phase shift keying version TG (SOQPSK-TG)) are of immediate interest.

The goal therefore is one involving single-carrier, constant-envelope modulation operating in a frequency selective channel. This is clearly an equalization and/or diversity problem. Diversity

Approved for public release; distribution is unlimited. 412TW-PA-14301 
reception, especially by widely separated antennas, is an obvious but expensive solution. Another feasible solution is transmit diversity when the size, weight and power constraints allow it. The most common scenario will be a system involving multiple transmit antennas with a single receive antenna, which can increase the reliability or throughput in multipath fading channels.

Under flat fading, if the transmitter knows the channels between each transmit and receive antenna, then spatio-temporal coding [3] is optimum in that it maximizes signal-to-noise ratio [2]. If the transmitter does not have this knowledge, then a diversity-maximizing orthogonal design (such as the Alamouti code [4]) is optimum [2]. Although OFDM is generally applied on a per subcarrier basis in frequency selective fading, it is not a viable approach under the constraints just described. Approaches suitable to single-carrier modulation are of interest, specifically the timereversed space-time block codes (TR-STBCs) [5]-[9]. It plays the role that the Alamouti code does in frequency non-selective fading with an uninformed transmitter. If the transmitter knows the channels, it is not obvious how it could use this channel state information since SOQPSK-TG does not have any "variable parameters" such as the number and location of constellation points. The only other variable available to the system is the power allocated to each transmit antenna.

In an effort to explore the robustness of transmit diversity in links prone to multipath fading, channel sounding experiments involving multiple transmit antennas have been conducted at Cairns Army Airfield, Ft. Rucker, Alabama, [10, 11] and Edwards Air Force Base, California, [12]. Initial experiments involving TR-STBC applied to the helicopter-to-ground channel sets revealed some curious behavior, which was described in [11] for QPSK. This same behavior with TR-STBC, SOQPSK-TG for the same data set is illustrated in Figure 1. This plot compares the simulated bit error rate (BER) performance of TR-STBC using minimum mean-squared error (MMSE) equalizers to the simulated BER performance of a link using only one of the two available channels. TR-STBC performs better than the single-channel link using only channel 2, but worse than the single-channel link using only channel 1. Clearly, channel 1 is better, in some sense, than channel 2. In fact, channel 1 is so much better than channel 2 that incorporating channel 2 into a TR-STBC system only makes things worse. These results imply that there are cases where it is better to abandon traditional TR-STBC and use only one of the two available channels. The fact that this curious behavior can occur on real channels prompts one to ask, "Can this curious behavior be derived from the given impulse responses of two channels?" In other words, it appears that with some form of channel state information, it is possible to achieve better performance than TR-STBC.

This paper answers the question. As a conceptual tool, we consider a fixed-power transmitter that allocates a portion of the fixed power to each channel. This power allocation is parameterized by $\rho$, the proportion of total power allocated to channel 1. Using only one of the two available channels is captured by the case $\rho=1$ (channel 1 only) or $\rho=0$ (channel 2 only). Next, we introduce Generalized TR-STBC (GTR-STBC), a modified version of TR-STBC that incorporates the unequal power allocation $\rho$. We also develop and analyze a MMSE equalizer that operates on the samples of SOQPSK-TG. By expressing the residual mean-squared error at the equalizer output as a function of $\rho$, we are able to choose $\rho$ to minimize the residual mean-squared error for a given pair of channel impulse responses. This criterion neatly captures the contributions of both ISI and additive noise at the equalizer output and it facilitates the derivation of mathematical expressions at the equalizer that are tractable. Also, generally speaking, reductions in mean-squared error lead 


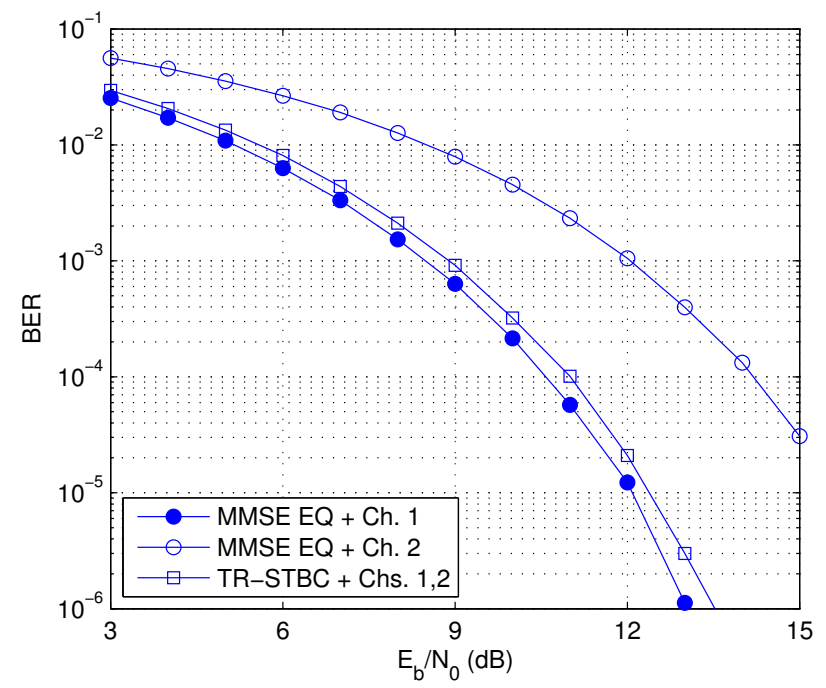

Figure 1: Simulated BER plots for 10-Mbit/s SOQPSK-TG using MMSE equalizers over a pair of representative impulse responses measured over a helicopter-to-ground channel. The circular markers are the BER results using a single antenna, the square markers are the BER results using both channels with TR-STBC.

to reductions in bit error.

Our numerical results show that the mean-squared error criterion is capable of identifying the cases where the curious behavior occurs. The conceptual tool of unequal power allocation together with GTR-STBC define a simple transmit diversity scheme based on the partial knowledge of the channel by the transmitter. Here, the transmitter only needs to know $\rho$, which is easy to compute at the receiver and send back to the transmitter. This simple scheme includes transmit selection diversity ( $\rho=0$ or 1$)$ and traditional TR-STBC $(\rho=1 / 2)$.

\section{MMSE EQUALIZATION OF SOQPSK-TG}

The system considered here is summarized in Figure 2. As before, the complex-valued baseband equivalent representation [13] is used for all signals. Starting with the block diagram of Figure 2 (a), the SOQPSK-TG signal $x_{c}(t)$ is transmitted through a channel impulse response $h_{c}(t)$ whose output, accompanied by thermal noise, forms the receive signal $r_{c}(t)$. After the application of an anti-aliasing low-pass filter with impulse response $h_{a}(t), T$-spaced samples of $r_{c}(t)$ are produced by an A/D converter. Assuming the anti-aliasing filter does not distort the received signal, the samples of the received signal may be expressed as

$$
r(n)=x(n) * h(n)+w(n)=\sum_{k=-L_{1}}^{L_{2}} h(k) x(n-k)+w(n),
$$

where

$$
r(n)=r_{c}(n T), \quad x(n)=x_{c}(n T), \quad h(n)=\left.h_{c}(t) * h_{a}(t)\right|_{t=n T} .
$$




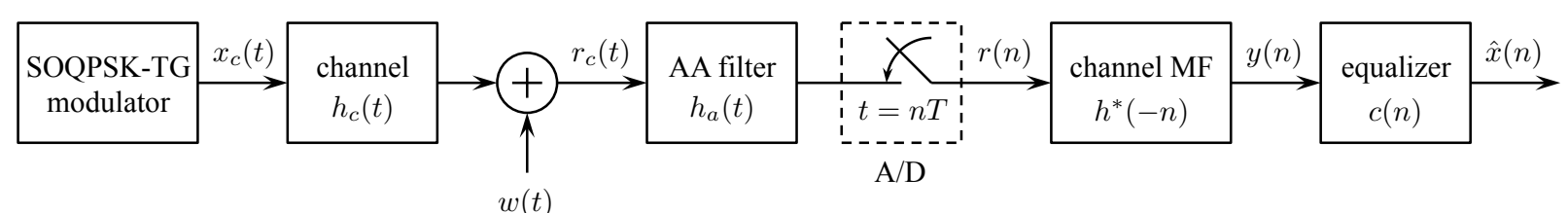

(a)

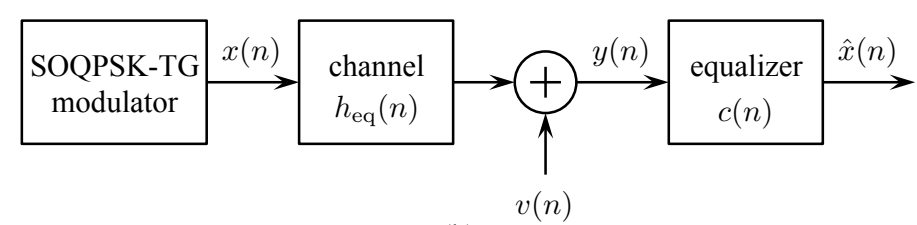

(b)

Figure 2: A block diagram of the system that applies an MMSE equalizer to samples of a received SOQPSK-TG signal: (a) the system showing the continuous-time signals, the anti-aliasing filter, and A/D converter; (b) the equivalent discrete-time system.

In (1) $w(n)$ is the $n$-th sample of a zero-mean complex-valued Gaussian random sequence with autocorrelation function

$$
R_{w}(k)=\frac{1}{2} \mathrm{E}\left\{w(n) w^{*}(n-k)\right\}=\sigma_{w}^{2} \delta(k) .
$$

Note that (1) assumes the discrete-time channel has support on $-L_{1} \leq n \leq L_{2}$. As a first step, we apply the samples $r(n)$ to a filter matched to the discrete-time channel to produce $y(n)$ :

$$
\begin{aligned}
y(n) & =r(n) * h^{*}(-n) \\
& =x(n) * \underbrace{h(n) * h^{*}(-n)}_{h_{\mathrm{eq}}(n)}+\underbrace{w(n) * h^{*}(-n)}_{v(n)} \\
& =\sum_{k=-L_{\mathrm{eq}}}^{L_{\mathrm{eq}}} h_{\mathrm{eq}}(k) x(n-k)+v(n),
\end{aligned}
$$

where $L_{\mathrm{eq}}=L_{1}+L_{2}$ and $v(n)$ is a complex valued Gaussian random sequence with zero mean and autocorrelation function

$$
R_{v}(k)=\frac{1}{2} \mathrm{E}\left\{v(n) v^{*}(n-k)\right\}=\sigma_{w}^{2} h_{\mathrm{eq}}(k) .
$$

The samples $y(n)$ form the input to an MMSE equalizer. The MMSE equalizer is an FIR filter with coefficients $c(n)$ for $-L_{c} \leq n \leq L_{c}$ designed to minimize the mean squared error between the equalizer filter output $\hat{x}(n)$ and the sequence $x(n)$. The entire system may be represented by an equivalent discrete-time system shown in Figure 2 (b).

The challenge with equalizing samples of the modulated signal is that the underlying continuoustime waveform is not wide-sense stationary [13]. This fact carries over to the samples of $x_{c}(t)$ and is captured by the fact that the autocorrelation function of the $x(n)$ is of the form

$$
R_{x}(k, \ell)=\frac{1}{2} \mathrm{E}\left\{x(k) x^{*}(\ell)\right\}
$$


that is, the autocorrelation function is a function of both sample indexes, not the difference between them. Consequently, the equalizer filter coefficients depend on sample index $n$. It is hard to see how this solution has any practical utility, especially in the presence of a real-time performance requirement. In the end, the designer is left with suboptimal approaches of reduced computational complexity whose accompanying performance penalty is acceptable.

The simplest suboptimal approach is to assume the signal samples are wide-sense stationary. Here, the autocorrelation function is of the form

$$
R_{x}(k-\ell)=\frac{1}{2} \mathrm{E}\left\{x(k) x^{*}(\ell)\right\}
$$

that is, the autocorrelation function depends on the difference of the sample time indexes. The wide-sense stationary assumption for $x(n)$ greatly simplifies the solution as the equalizer coefficients no longer depend on the samples index $n$.

The question is now, what function should be used for the autocorrelation function $R_{x}(k)$ ? Two approximations are investigated in [17]. The first is an empirically-derived autocorrelation function which is obtained by generating a large number of samples $x(n)$ and using the standard estimation technique assuming wide sense stationarity. Given $L$ samples of $x(n)$ for $n=0,1, \ldots, L-1$, this empirical autocorrelation function is

$$
R_{\mathrm{e}}(k)=\frac{1}{2(L-k)} \sum_{n=k}^{L-1} x(n) x^{*}(n-k), \quad 0 \leq k<L-1
$$

together with

$$
R_{\mathrm{e}}(k)=R_{\mathrm{e}}^{*}(-k), \quad-L<k<0 .
$$

The second approximation is to assume the data are uncorrelated which generates a correlation function of the form

$$
R_{\mathrm{i}}(k)=\sigma_{x}^{2} \delta(k)
$$

In [17], we demonstrated that MMSE equalizers based on above approximations exhibit almost identical performance. Consequently, we use the second approximation as this choice simplifies the computations of the equalizer filter coefficients.

Because the optimum equalizer coefficients no longer depend on the samples index $n$, the relationship between $x(n)$ and the equalizer output $\hat{x}(n)$ is

$$
\hat{x}(n)=c(n) * y(n)=\sum_{k=-L_{c}}^{L_{c}} c(k) y(n-k) .
$$

The vector of filter coefficients that minimizes the mean squared error

$$
\mathcal{E}=\mathrm{E}\left\{|x(n)-\hat{x}(n)|^{2}\right\}
$$


is given by

$$
\mathbf{c}=\left[\mathbf{G G}^{\dagger}+\frac{2}{E_{b} / N_{0}} \mathbf{H}_{\mathrm{eq}}\right]^{-1} \mathbf{g}^{\dagger}
$$

where $\mathbf{c}$ is the $\left(2 L_{c}+1\right) \times 1$ vector of filter coefficients, $\mathbf{G}$ is the $\left(2 L_{c}+1\right) \times\left(2 L_{c}+1+2 L_{\text {eq }}\right)$ matrix

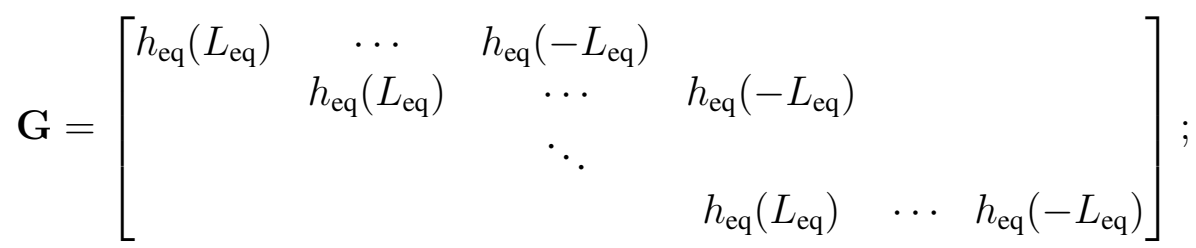

$\mathbf{H}_{\mathrm{eq}}$ is the $\left(2 L_{c}+1\right) \times\left(2 L_{c}+1\right)$ matrix given by

$$
\mathbf{H}_{\mathrm{eq}}=\left[\begin{array}{ccc}
h_{\mathrm{eq}}(0) & \cdots & h_{\mathrm{eq}}\left(-2 L_{c}\right) \\
\vdots & & \vdots \\
h_{\mathrm{eq}}\left(2 L_{c}\right) & \cdots & h_{\mathrm{eq}}(0)
\end{array}\right],
$$

where it is understood that $h_{\mathrm{eq}}(k)=0$ for $|k|>L_{\mathrm{eq}}$.

Vector $\mathbf{g}$ is the $1 \times\left(2 L_{c}+1\right)$ vector given by

$$
\mathbf{g}=\left[\begin{array}{lll}
\mathbf{0}_{1 \times\left(L_{c}-L_{\mathrm{eq}}\right)} & \mathbf{h}_{\mathrm{eq}} & \mathbf{0}_{1 \times\left(L_{c}-L_{\mathrm{eq}}\right)}
\end{array}\right],
$$

where $0_{1 \times\left(L_{c}-L_{\text {eq }}\right)}$ is a row vector comprising $L_{c}-L_{\text {eq }}$ zeros (here we assume $L_{c}>L_{\text {eq }}$, i.e., the equalizer is longer than the channel), and $\mathbf{h}_{\mathrm{eq}}$ is the $1 \times\left(2 L_{\mathrm{eq}}+1\right)$ vector given by

$$
\mathbf{h}_{\mathrm{eq}}=\left[\begin{array}{lllll}
h_{\mathrm{eq}}\left(L_{\mathrm{eq}}\right) & \cdots & h_{\mathrm{eq}}(0) & \cdots & h_{\mathrm{eq}}\left(-L_{\mathrm{eq}}\right)
\end{array}\right] .
$$

The corresponding mean squared error is

$$
\mathcal{E}_{\min }=\sigma_{x}^{2}\left(1-\mathbf{g}\left[\mathbf{G G}^{\dagger}+\frac{2}{E_{b} / N_{0}} \mathbf{H}_{\mathrm{eq}}\right]^{-1} \mathbf{g}^{\dagger}\right) .
$$

\section{GENERALIZED TR-STBC (GTR-STBC): NON-EQUAL POWER ALLOCATIONS USING TR-STBC}

An abstraction (to the sample level) for a $2 \times 1$ TR-STBC system is illustrated in Figure 3. Here the system transmits $2 L$ samples of an SOQPSK-TG signal sampled at $N$ samples/bit over two transmit antennas to one receive antenna. The equivalent discrete-time channel between transmit antenna 1 and the receive antenna is represented by the impulse response $h_{1}(n)$ for $-L_{1,1} \leq$ $n \leq L_{1,2}$ whereas the equivalent discrete-time channel between transmit antenna 2 and the receive antenna is represented by the impulse response $h_{2}(n)$ for $-L_{2,1} \leq n \leq L_{2,2}$. 


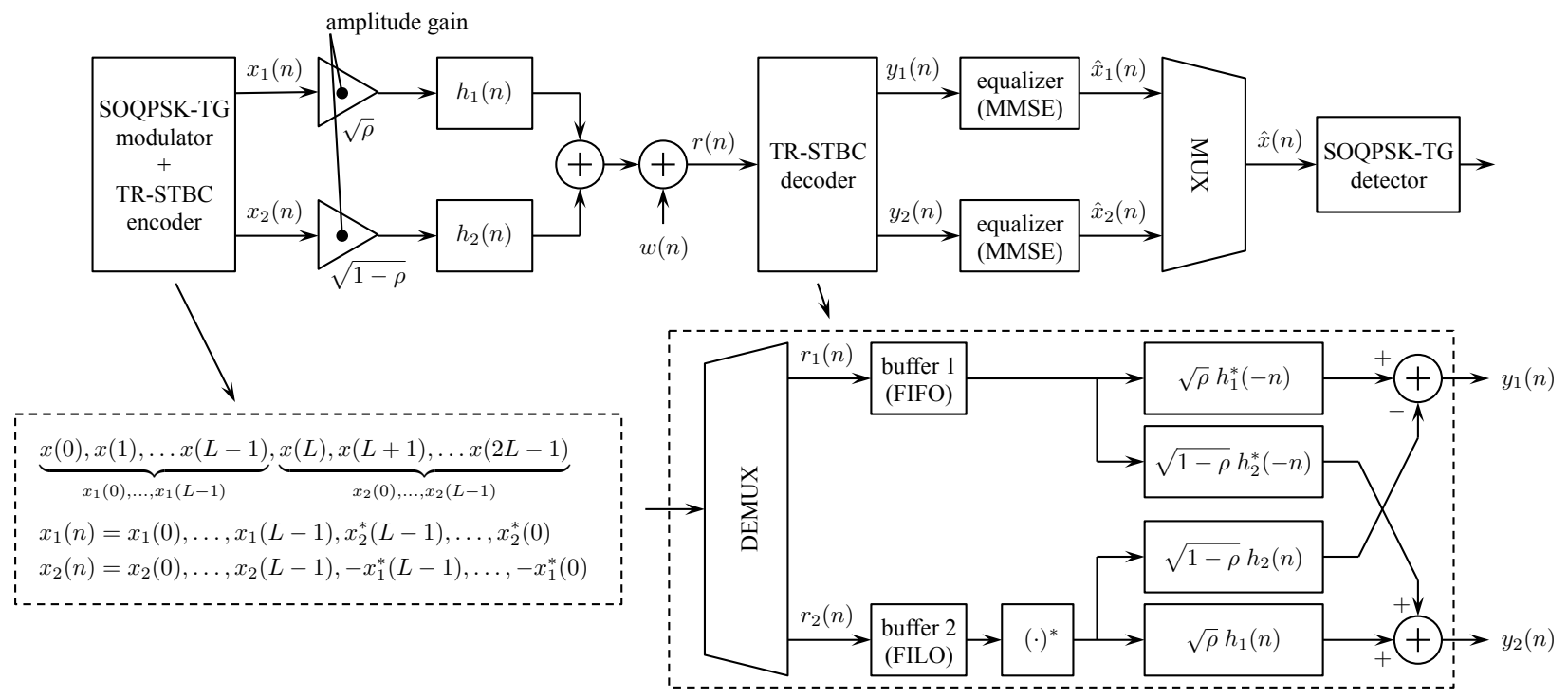

Figure 3: A block diagram of the TR-STBC system based on unequal power allocation using $0 \leq \rho \leq 1$.

The TR-STBC encoder partitions the sample sequence $x(0), \ldots, x(2 L-1)$ into two sequences $x_{1}(n)$ and $x_{2}(n)$ as shown in Figure 3. The length- $2 L$ packet is transmitted in two intervals ${ }^{1}$ each spanning $L$ sample intervals. During the first interval $x_{1}(0), \ldots, x_{1}(L-1)$ is transmitted from antenna 1 whereas $x_{2}(0), \ldots, x_{2}(L-1)$ is transmitted from antenna 2. During the second interval, $x_{2}^{*}(L-1), \ldots, x_{2}^{*}(0)$ is transmitted from antenna 1 whereas $-x_{1}^{*}(L-1), \ldots,-x_{1}^{*}(0)$ is transmitted from antenna 2.

Power division using $0 \leq \rho \leq 1$ is accomplished by modified the TR-STBC system along the lines illustrated in Figure 3. Amplitude scaling is applied to the signals entering each channel so as to divide the power between the channels. Here $\rho$ represents the proportion of total power allocated to transmit antenna 1 . The traditional TR-STBC system ${ }^{2}$ is a special case for which $\rho=1 / 2$. The square-root is used in Figure 3 because the amplitudes are what are being modified-the energy (or power) is the square of the amplitude.

The received signal $r(n)$ is given by

$$
r(n)=\sqrt{\rho} x_{1}(n) * h_{1}(n)+\sqrt{1-\rho} x_{2}(n) * h_{2}(n)+w(n),
$$

where $w(n)$ is a complex-valued Gaussian random sequence with zero mean and autocovariance function given by (2). The TR-STBC decoder partitions $r(n)$ into $r_{1}(n)$ and $r_{2}(n)$ as follows:

$$
\begin{aligned}
r_{1}(n) & =r(n) \text { for } 0 \leq n \leq L-1 \\
r_{2}(n-L) & =r(n) \text { for } L \leq n \leq 2 L-1 .
\end{aligned}
$$

\footnotetext{
${ }^{1}$ In a practical implementation, a guard interval at least as long as the longest channel impulse response must be inserted between the two intervals. Here, such an interval is assumed, although we won't complicate the notation to make this explicit.

${ }^{2}$ In the traditional TR-STBC system, $\rho=1 / 2$ is included in neither the development nor the notation because the same power is assumed to be applied to each channel. Hence there is no need to account for it, other than in normalizing the noise variance.
} 
These two sequences are given by

$$
\begin{aligned}
& r_{1}(n)=\sqrt{\rho} x_{1}(n) * h_{1}(n)+\sqrt{1-\rho} x_{2}(n) * h_{2}(n)+w_{1}(n) \\
& r_{2}(n)=\sqrt{\rho} x_{2}^{*}(-n) * h_{1}(n)-\sqrt{1-\rho} x_{1}^{*}(-n) * h_{2}(n)+w_{2}(n),
\end{aligned}
$$

where $w_{1}(n)$ and $w_{2}(n)$ are related to $w(n)$ the same way $r_{1}(n)$ and $r_{2}(n)$ are related to $r(n)$. The TR-STBC decoder processes $r_{1}(n)$ and $r_{2}(n)$ using a bank of filters based on the channel impulse responses $h_{1}(n)$ and $h_{2}(n)$ as shown. The result of this processing is a pair of parallel sequences $y_{1}(n)$ and $y_{2}(n)$ which may be expressed as

$$
\begin{aligned}
& y_{1}(n)=r_{1}(n) * \sqrt{\rho} h_{1}^{*}(-n)-r_{2}^{*}(-n) * \sqrt{1-\rho} h_{2}(n) \\
&=x_{1}(n) * \underbrace{\left[\rho h_{1}(n) * h_{1}^{*}(-n)+(1-\rho) h_{2}(n) * h_{2}^{*}(-n)\right]}_{h_{\mathrm{eq}}(n)} \\
&+\underbrace{w_{1}(n) * \sqrt{\rho} h_{1}^{*}(-n)+w_{2}^{*}(-n) * \sqrt{1-\rho} h_{2}(n)}_{v_{1}(n)}
\end{aligned}
$$

and

$$
\begin{aligned}
& y_{2}(n)=r_{1}(n) * \sqrt{1-\rho} h_{2}^{*}(-n)+r_{2}^{*}(-n) * \sqrt{\rho} h_{1}(n) \\
&=x_{2}(n) * \underbrace{\left[(1-\rho) h_{2}(n) * h_{2}^{*}(-n)+\rho h_{1}^{*}(-n) * h_{1}(n)\right]}_{h_{\mathrm{eq}}(n)} \\
&+\underbrace{\sqrt{1-\rho} w_{1}(n) * h_{2}^{*}(-n)+\sqrt{\rho} w_{2}^{*}(-n) * h_{1}(n)}_{v_{2}(n)} .
\end{aligned}
$$

These equations show that the equivalent composite channel for non-equal power allocation is

$$
h_{\mathrm{eq}}(n)=\rho \underbrace{h_{1}(n) * h_{1}^{*}(-n)}_{\eta_{1}(n)}+(1-\rho) \underbrace{h_{2}(n) * h_{2}^{*}(-n)}_{\eta_{2}(n)} \text {. }
$$

Because the support for $h_{1}(n)$ is $-L_{1,1} \leq n \leq L_{1,2}$, the support for $\eta_{1}(n)$ is $-\left(L_{1,1}+L_{1,2}\right) \leq n \leq$ $\left(L_{1,1}+L_{1,2}\right)$. Similarly because the support for $h_{2}(n)$ is $-L_{2,1} \leq n \leq L_{2,2}$, the support for $\eta_{2}(n)$ is $-\left(L_{2,1}+L_{2,2}\right) \leq n \leq\left(L_{2,1}+L_{2,2}\right)$. Consequently, the support for $h_{\mathrm{eq}}(n)$ is $-L_{\mathrm{eq}} \leq n \leq L_{\mathrm{eq}}$ where

$$
L_{\text {eq }}=\max \left\{L_{1,1}+L_{1,2}, L_{2,1}+L_{2,2}\right\} .
$$

The noise sequences $v_{1}(n)$ and $v_{2}(n)$ are complex-valued Gaussian random sequences each with zero mean and autocorrelation and cross correlation functions

$$
\begin{gathered}
\frac{1}{2} \mathrm{E}\left\{v_{1}(n) v_{1}^{*}(n-k)\right\}=\frac{1}{2} \mathrm{E}\left\{v_{2}(n) v_{2}^{*}(n-k)\right\}=\sigma_{w}^{2} h_{\mathrm{eq}}(k) . \\
\mathrm{E}\left\{v_{1}(n) v_{2}^{*}(n-k)\right\}=0 .
\end{gathered}
$$


By way of summary, the TR-STBC system presents to the equalizers the sequences $y_{1}(n)$ and $y_{2}(n)$ which may be represented by

$$
\begin{aligned}
& y_{1}(n)=x_{1}(n) * h_{\mathrm{eq}}(n)+v_{1}(n) \\
& y_{2}(n)=x_{2}(n) * h_{\mathrm{eq}}(n)+v_{2}(n)
\end{aligned}
$$

where $h_{\mathrm{eq}}(n)$ is given by (25). The noise terms $v_{1}(n)$ and $v_{2}(n)$ are uncorrelated zero-mean Gaussian random sequences each with autocorrelation function (27). A pair of equalizers operate in parallel on $y_{1}(n)$ and $y_{2}(n)$. Because the the noise sequences $v_{1}(n)$ and $v_{2}(n)$ are statistically equivalent and $h_{\mathrm{eq}}(n)$ is common to both, the pair of equalizers operating on $y_{1}(n)$ and $y_{2}(n)$ are identical as long as $a_{1}(n)$ and $a_{2}(n)$ are statistically equivalent (the usual case). Any equalizer can be applied here (linear or non-linear, with or without noise whitening) with the usual performancecomplexity tradeoffs. Here, we apply the approximate MMSE equalizer introduced in previous section because the MMSE equalizer permit a mathematically tractable analysis for the resulting mean-squared error. We leverage the analytical expression to find the value of $\rho$ that minimizes the mean squared error.

The equalizer filters of Figure 3 are identical, and the vector of filter coefficients is given by (13) and the corresponding mean squared error is given by (18). In these equations, $\mathbf{G}, \mathbf{g}$, and $\mathbf{H}_{\mathrm{eq}}$ may be expressed in terms of power allocation parameter $\rho$. Using (25), it is straightforward to show that

$$
\begin{aligned}
\mathbf{G} & =\rho \mathbf{G}_{1}+(1-\rho) \mathbf{G}_{2} \\
\mathbf{g} & =\rho \mathbf{g}_{1}+(1-\rho) \mathbf{g}_{2} \\
\mathbf{H}_{\mathrm{eq}} & =\rho \mathbf{H}_{1}+(1-\rho) \mathbf{H}_{2}
\end{aligned}
$$

where $\mathbf{G}_{1}, \mathbf{g}_{1}$, and $\mathbf{H}_{1}$ are formed from $\eta_{1}(n)$ the same way $\mathbf{G}, \mathbf{g}$, and $\mathbf{H}_{\text {eq }}$ are formed from $h_{\mathrm{eq}}(n)$, respectively. Similar definitions apply to $\mathbf{G}_{2}, \mathbf{g}_{2}$, and $\mathbf{H}_{2}$ with $\eta_{2}(n)$. Making the substitutions for $\mathbf{G}, \mathbf{g}$, and $\mathbf{H}_{\mathrm{eq}}$ gives

$$
\mathbf{c}=\mathbf{M}^{-1}\left(\rho \mathbf{g}_{1}+(1-\rho) \mathbf{g}_{2}\right)^{\dagger}
$$

and

$$
\mathcal{E}_{\min }=\sigma_{x}^{2}\left[1-\left(\rho \mathbf{g}_{1}+(1-\rho) \mathbf{g}_{2}\right) \mathbf{M}^{-1}\left(\rho \mathbf{g}_{1}+(1-\rho) \mathbf{g}_{2}\right)^{\dagger}\right]
$$

where

$$
\mathbf{M}=\left(\rho \mathbf{G}_{1}+(1-\rho) \mathbf{G}_{2}\right)\left(\rho \mathbf{G}_{1}+(1-\rho) \mathbf{G}_{2}\right)^{\dagger}+\frac{2}{E_{b} / N_{0}}\left(\rho \mathbf{H}_{1}+(1-\rho) \mathbf{H}_{2}\right) .
$$

Equation (35) is the desired relationship: for a given pair of channels $h_{1}(n)$ and $h_{2}(n)$, it expresses the mean squared error at the output of the MMSE equalizer as a function of the power allocation $\rho$. Thus, for a fixed pair channels, one can choose the power allocation to minimize the achievable mean-squared error. 


\section{NUMERICAL RESULTS}

The forgoing analysis was applied to a helicopter-to-ground radio link using 190,716 channel impulse responses from the Black Mountain flight path at Edwards AFB, California, captured during the channel sounding experiment described in [10]. For the modulation, we assume $10 \mathrm{Mbit} / \mathrm{s}$ SOQPSK-TG with the equalizers operating at 2 samples/bit. In the results shown below, $h_{1}(n)$ is the equivalent discrete-time channel between the nose antenna and the receive antenna and $h_{2}(n)$ is the channel between the tail antenna and the receive antenna.

Two normalizations are applied to the channels: the natural normalization and the equal-energy normalization. Let $h_{1, \mathrm{u}}(n)$ and $h_{2, \mathrm{u}}(n)$ be unnormalized channel impulse responses for the two equivalent discrete-time channels obtained directly from the channel sounding data, and let

$$
E_{1}=\sum_{n=-L_{1,1}}^{L_{1,2}}\left|h_{1, \mathrm{u}}(n)\right|^{2} \quad E_{2}=\sum_{n=-L_{2,1}}^{L_{2,2}}\left|h_{2, \mathrm{u}}(n)\right|^{2}
$$

be the energies in two channels. The natural normalization uses

$$
h_{1}(n)=\frac{1}{\sqrt{E}} h_{1, \mathrm{u}}(n) \quad h_{2}(n)=\frac{1}{\sqrt{E}} h_{2, \mathrm{u}}(n)
$$

where $E=\max \left\{E_{1}, E_{2}\right\}$. This normalizes the stronger of the two channels to unit energy. ${ }^{3}$ We call this the natural normalization because in real multi-antenna scenarios, it is often the case that one of the channels is stronger than the other.

For the equal-energy normalization, we use

$$
h_{1}(n)=\frac{1}{\sqrt{E_{1}}} h_{1, \mathrm{u}}(n) \quad h_{2}(n)=\frac{1}{\sqrt{E_{2}}} h_{2, \mathrm{u}}(n) .
$$

Here, both channels are normalized to unit energy. This is more typical of statistical or mathematical models of multi-antenna propagation.

The numerical results were produced as follows. For each of the 190,716 pairs of channel impulse responses, the impulse responses were normalized using one of the two procedures described above. The value of $\rho$ that minimizes (35) for $L_{c}=5 \times L_{\mathrm{eq}}$ [18] was computed. The results corresponding to the natural normalization are shown in Figure 4. Here, the ISI is such that the equalizer is able to reduce the residual ISI to the point where signal-to-noise ratio is the dominant factor. The end result is that the ISI, signal-to-noise ratio, and power allocation tradeoff becomes mostly a tradeoff between signal-to-noise ratio and power allocation. This tradeoff is such that the mean squared error is minimized by applying $100 \%$ of the available power to the lower antenna [corresponding to $\left.h_{2}(n)\right]$ in the vast majority of the cases $\left(152,246\right.$ or $80 \%$ for $E_{b} / N_{0}=10 \mathrm{~dB}$, 130,989 or $69 \%$ for $E_{b} / N_{0}=20 \mathrm{~dB}$ ). With the equal-energy normalization, the signal-to-noise ratio penalty associated with $h_{1}(n)$ is removed and the tradeoff reduces to an ISI and power allocation tradeoff. In this case, summarized in Figure 5, the situation reverses. The mean squared error is minimized by applying $100 \%$ of the available power to the upper antenna in the vast majority of the cases $\left(147,738\right.$ or $77 \%$ for $E_{b} / N_{0}=10 \mathrm{~dB}, 155,786$ or $82 \%$ for $\left.E_{b} / N_{0}=20 \mathrm{~dB}\right)$.

\footnotetext{
${ }^{3}$ The motivation for unit energy is for scaling the noise variance to define the signal-to-noise ratio.
} 

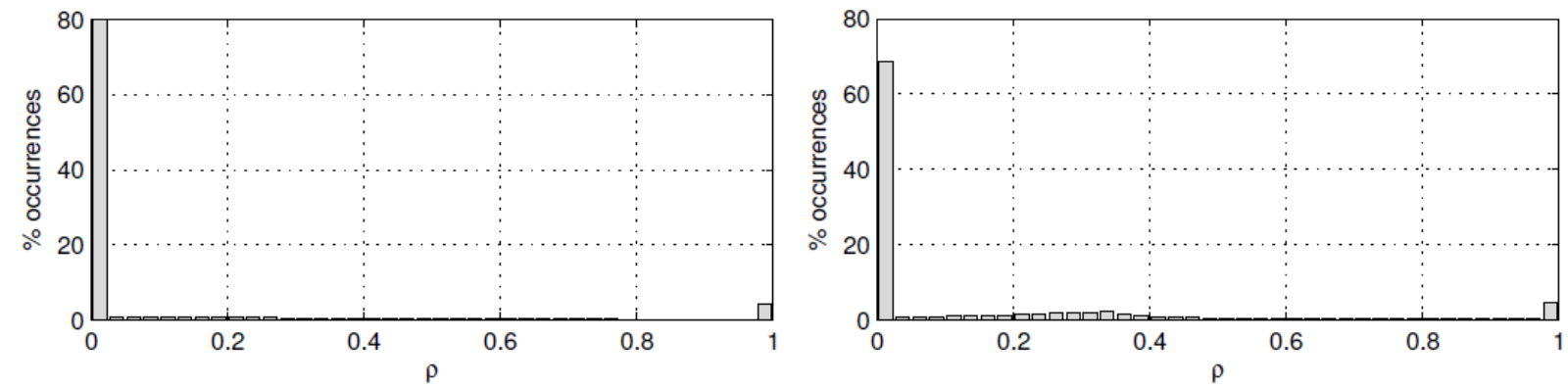

Figure 4: Optimum power allocations in the mean-squared error sense for the measured channel impulse responses from the Black Mountain flight path at Edwards AFB, California, using the natural normalization: (left) $E_{b} / N_{0}=10 \mathrm{~dB}$; (right) $E_{b} / N_{0}=20 \mathrm{~dB}$.
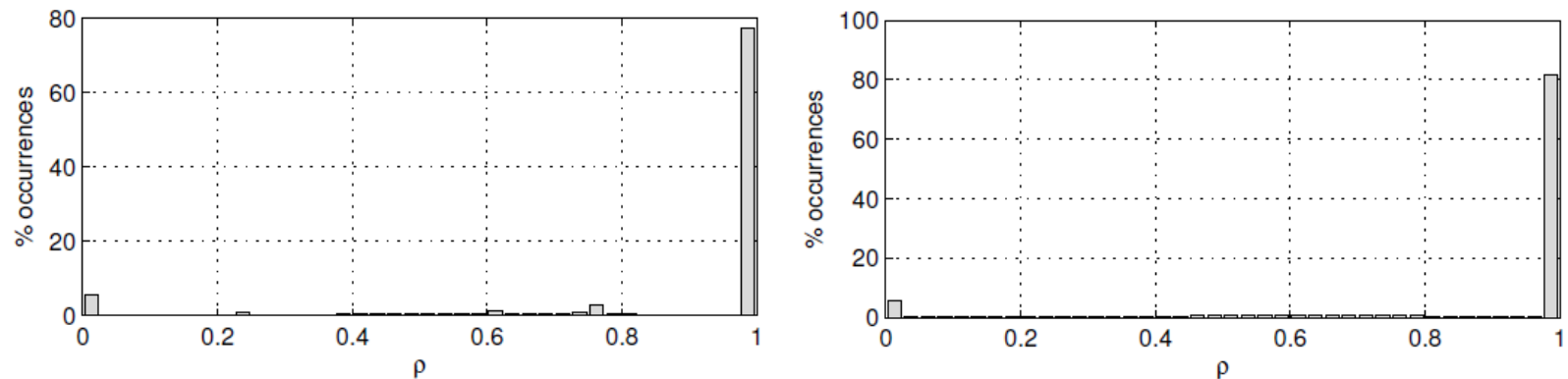

Figure 5: Optimum power allocations in the mean-squared error sense for the measured channel impulse responses from the Black Mountain flight path at Edwards AFB, California, using the equal-energy normalization: (left) $E_{b} / N_{0}=10 \mathrm{~dB}$; (right) $E_{b} / N_{0}=20 \mathrm{~dB}$. 

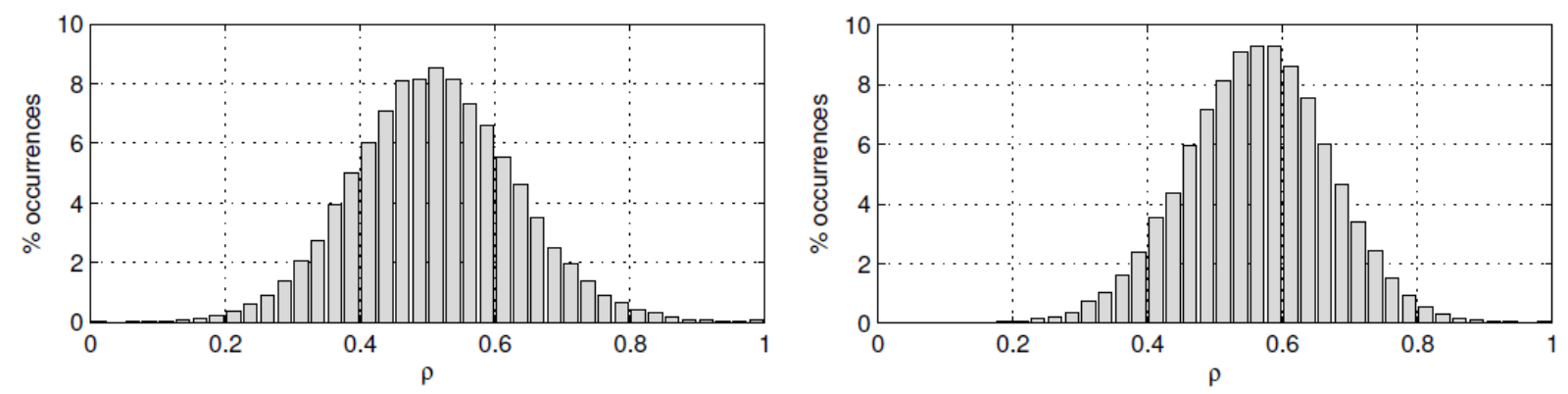

Figure 6: Optimum power allocations in the mean-squared error sense for pairs of equal-energy channels where the channel coefficients are i.i.d. zero-mean complex-valued Gaussian random variables: (left) $E_{b} / N_{0}=10 \mathrm{~dB}$; (right) $E_{b} / N_{0}=20 \mathrm{~dB}$.

The channel between the upper antenna and the receiver, $h_{1}(n)$, is characterized by lower signalto-noise ratio and lower ISI than $h_{2}(n)$, the channel between the lower antenna and the receiver. Using the natural normalization, the equalizer is able to remove ISI to the point where signal-tonoise ratio is the dominant factor. In this case applying $100 \%$ of the available energy to the lower antenna is the optimum approach in most of the cases. Using the equal-energy normalization, differences in signal-to-noise ratio are removed so that ISI becomes the dominant factor. Because the ISI on the channel from the upper antenna is so much less than the ISI from the lower antenna, applying $100 \%$ of the available energy to the upper antenna is the optimum.

It is interesting to compare these results with what might be inferred from using a simple statistical channel model. To do so, we use a simple Gaussian model for each channel such as that used in [19]. In this experiment, channel 1 comprises 3 independent and identically distributed (i.i.d.) zero-mean complex-valued Gaussian random variables and channel 2 comprises 6 i.i.d. zero-mean complex-valued Gaussian random variables. These numbers, 3 and 6, are the average lengths of $h_{1}(n)$ and $h_{2}(n)$, respectively, in our measured data set. The channels were normalized using the equal-energy normalization described above and 190,716 independent realizations were produced. The results are summarized by the histograms in Figure 6 . The temptation is to think of the optimum $\rho$ as a normally distributed random variable, but it should be kept in mind that this is not the case because $0 \leq \rho \leq 1$. For modest values of $E_{b} / N_{0}$ we observe that the mean value of the optimum $\rho$ is about 0.5 . Given the fact that $\rho=0.5$ corresponds to traditional TR-STBC, we see that the simple statistical model suggests that traditional TR-STBC is the best on average. This is in contrast to the conclusion drawn from the measured channel data, where a strong preference for transmit selection diversity is observed.

As $E_{b} / N_{0}$ increases, the optimum value of $\rho$ appears to increase. This is explained as follows: as $E_{b} / N_{0}$ increases, the contribution to squared error from additive noise is reduced and ISI starts to become the dominant contributor. The optimum $\rho>0.5$ means the system prefers to allocate more energy to channel 1 than channel 2 . This makes sense because channel 1 is shorter, and this tends to contribute less residual ISI at the equalizer output. 


\section{CONCLUSIONS}

A criterion that allows one to predict when it is better to use transmit selection diversity (i.e., one transmit antenna) or the diversity achievable through TR-STBC (i.e., two transmit antennas) was developed. The criterion is the residual mean-squared error at the output of an MMSE equalizer. The residual mean-squared error was not only a mathematically tractable quantity, but also an excellent predictor of the curious behavior illustrated in Figure 1.

The above criterion was applied to a set of measured channel impulse responses collected from the Black Mountain flight path at Edwards AFB, California. For each pair of channel impulse responses, the value of $\rho$ that minimized the residual mean-squared error (35) was computed and used to form histograms to summarize the results. Transmit selection diversity (i.e. applying all of the available power to the stronger channel) is optimum when two channels have unequal gains in a 2-transmit, 1-receive antenna system operating in a frequency non-selective fading environment. In contrast, we concluded that on a frequency selective fading channel, the optimum approach was to apply power to produce the best trade-off between signal-to-noise ratio and ISI. The optimum value of $\rho$ associated with the GTR-STBC system described in this paper identified this trade-off. There were some channel pairs for which $\rho=0$ or 1 was the optimum (transmit diversity case) and some channel pairs for which $\rho=1 / 2$ (traditional TR-STBC). But there were many channel pairs for which neither of these was optimum.

The results on our measured channels also indicated that transmit selection diversity was more common than traditional TR-STBC. On a statistical channel, such as the one used in [19], traditional TR-STBC is the best thing to do on average. This is in contrast to the results from the measured channels. Consequently, the optimum power allocation in a real setting is not predicted well by simple statistical channel models.

\section{ACKNOWLEDGEMENTS}

This work was funded by the Test Resource Management Center (TRMC) Test and Evaluation Science and Technology (T\&E/S\&T) Program through the U.S. Army Program Executive Office for Simulation, Training and Instrumentation (PEO STRI) under contract W900KK-09-C-0016 (M4A) and W900KK-13-C-0026 (PAQ).

\section{REFERENCES}

[1] IRIG Standard 106-04: Telemetry Standards, Range Commanders Council Telemetry Group, Range Commanders Council, White Sands Missile Range, New Mexico, 2004, (Available online at http://www.wsmr.army.mil/RCCsite/Pages/Publications.aspx).

[2] D. Tse and P. Viswanath, Fundamentals of Wireless Communication. Cambridge, UK: Cambridge University Press, 2005.

[3] G. Raleigh and J. Cioffi, "Spatio-temporal coding for wireless communications," IEEE Transactions on Communications, vol. 46, no. 3, pp. 357-366, March 1998. 
[4] S. Alamouti, "A simple transmit diversity technique for wireless communications," IEEE Journal on Selected Areas in Communications, vol. 16, no. 8, pp. 1451-1458, October 1998.

[5] E. Lindskog and A. Paulraj, "A transmit diversity scheme for channels with intersymbol interference," in Proceedings of the IEEE International Conference on Communications, New Orleans, June 2000.

[6] N. Al-Dhahir, A. Naguib, and R. Calderbank, "Finite-length MIMO decision feedback equalization for space-time block-coded signals over multipath-fading channels," IEEE Transactions on Vehicular Technology, vol. 50, no. 4, pp. 1176-1182, July 2001.

[7] S. Diggavi, N. Al-Dhahir, and R. Calderbank, "Algebraic properties of spacetime block codes in intersymbol interference multiple-access channels," IEEE Transactions on Information Theory, vol. 49, no. 10, pp. 2403-2414, October 2003.

[8] W. Gerstacker, F. Obernosterer, R. Schober, A. Lehmann, and L. Lampe, "Equalization concepts for Alamouti's space-time block code," IEEE Transactions on Communications, vol. 52, pp. 1178-1190, July 2004.

[9] Y. Zhu and K. Letaief, "Single-carrier frequency-domain equalization with decision-feedback processing for time-reversal space-time block-coded systems," IEEE Transactions on Communications, vol. 53, no. 7, pp. 1127-1131, July 2005.

[10] M. Rice and M. Jensen, "Mulitpath propagation for helicopter-to-ground MIMO links," in Proceedings of the IEEE Military Communications Conference, Baltimore, MD, October 2011.

[11] M. Rice and M. Saquib, "MIMO equalization for helicopter-to-ground communications," in Proceedings of the IEEE Military Communications Conference, Baltimore, MD, October 2011.

[12] M. Rice and M. Jensen, "A comparison of L-band and C-band multipath propagation at Edwards AFB," in Proceedings of the International Telemetering Conference, Las Vegas, NV, October 2011.

[13] J. Proakis and M. Salehi, Digital Communications, 5th ed. New York: McGraw-Hill, 2007.

[14] L. Li and M. K. Simon, "Performance of coded OQPSK and MIL-STD SOQPSK with iterative decoding," IEEE Transactions on Communications, vol. 52, pp. 1890-1900, November 2004.

[15] T. J. Hill, "A nonproprietary, constant envelope, variant of shaped offset QPSK (SOQPSK) for improved spectral containment and detection efficiency," in Proceedings of the IEEE Military Communications Conference, vol. 1, Los Angeles, CA, October 2000, pp. 347-352.

[16] J. B. Anderson, T. Aulin, and C.-E. Sundberg, Digital Phase Modulation. New York: Plenum Press, 1986. 
[17] M. Rice, M. S. Afran, and M. Saquib, "MMSE equalization for aeronautical telemetry channels," in Proceedings of the International Telemetering Conference, San Diego, CA, October 2014.

[18] J. Treichler, I. Fijalkow, and C. Johnson, "Fractionally spaced equalizers: How long should they be?" IEEE Signal Processing Magazine, May 1996.

[19] Z. Zhang, T. Duman, and E. Kurtas, "Achievable information rates and coding for MIMO systems over ISI channels and frequency-selective fading channels," IEEE Transactions on Communications, vol. 52, no. 10, pp. 1698-1710, October 2004. 